\title{
Managing a local PDS scheme
}

\author{
A qualitative analysis of the planning, implementation and management of a PDS scheme: lessons for \\ commissioning of dental services J. T. Newton, B. Alexandrou, B. Dixon Bate and H. Best Br Dent J 2006;
} 200: 625-630

\section{Aim}

To identify the experiences of primary care trust employees, the dental teams and other key individuals of the planning, implementation and management of a Personal Dental Services scheme.

Method

A thematic analysis of a series of qualitative interviews with 29 individuals who were involved in the planning, implementation and management of a PDS scheme in South East London.

Findings

Nine key themes were analysed from the data. For each theme perspectives could be identified for both the employees of the primary care trusts and the dental team. These perspectives differ in key respects. Conclusions

Practitioners value the PDS scheme and consider it a positive experience. They suggest that it has led to an increase in quality of care, and a more professional management approach to the practice. The practice team felt that they have benefited from an enhanced working environment. The main concern expressed was that patient registrations were not being accurately assessed. Those involved in the management of the PDS scheme, while endorsing local commissioning arrangements, were concerned that it was not known whether PDS was meeting local needs. There was little quality benchmarking, which would have allowed robust measure of success. The contract model and outcomes should have been more sensitively designed. There was concern expressed that the small number of practices who participated in the pilot scheme prohibits the possibility of thoroughly analysing the impact of local commissioning. Future local commissioning should identify mechanisms for ensuring the effective planning, management and evaluation of the impact of the schemes. A core element of this will be the specification of appropriate goals for commissioning.

\section{IN BRIEF}

- Dental practitioners within Phase 1 Personal Dental Services Pilot schemes felt that their participation was valuable and positive.

- Practitioners perceive that the PDS pilot scheme allowed them to improve the quality of care they delivered to patients and the standard of management within the practice.

- Benchmarking performance and ensuring that mechanisms for monitoring and improving the quality of service were felt to be important components to the development of new systems of remuneration, but had been largely overlooked in the pilot schemes.

- Local commissioning should identify mechanisms for ensuring the effective planning, management and evaluation of changes in remuneration.

\section{COMMENT}

This is a comprehensive piece of research that complements the earlier national evaluation of the PDS scheme. It gives further insight into the complexities involved in the negotiation of changes to dental services from the perspective of those responsible for planning and implementing the change and from those responsible for carrying out the changes.

Further changes to the delivery of dental services will require careful management. Participants in this study have been shown to have different interpretations of the changes related to the PDS scheme, which was most apparent when they considered the aim of the scheme. This is likely to have implications on all stages of the management and implementation of the scheme as the outcome measures should reflect the aims of the scheme.

The management participants considered that although the PDS scheme offered dentists financial stability, difficulties existed with the scheme. This was due to the lack of quality benchmarks that would enable performance monitoring to show improvements in either patient numbers or quality of care. There were also concerns that capitation may become the replacement 'treadmill' with GDPs focusing on registration numbers.

The dentists were positive about the PDS scheme as it enabled them to focus on quality, improved staff morale and offered financial stability. Problems were encountered with the IT systems and the time required developing a business model. There were concerns that evaluations were inappropriately focused on patient registration especially as it was felt that the DPB were unable to provide accurate data on registrations.

The findings of this research have implications on current contract negotiations that are being held between the PCTs and GDPs. It suggests that the development of systems for monitoring that are relevant to the aims of the PDS scheme have been neglected due to the pressure to meet tight deadlines imposed by the scheme. It poses an interesting challenge to all those involved in local commissioning suggesting that efforts must be made to ensure that both the PCT and GDPs have a similar understanding of the aims and objectives of the new ways of working. The development of appropriate monitoring and evaluation systems are required for the new dental contract with a greater focus on the aims of the changes, with realistic goals, rather than the time constraints imposed by policy.

W. Baird, Lecturer Health Services Research, Trent RDSU, School of Health and Related Research, University of Sheffield DOI: 10.1038/sj.bdj.4813646 